\title{
Transparent, Superflexible Doubly Cross-Linked Polyvinylpolymethylsiloxane Aerogel Superinsulators via Ambient Pressure Drying
}

\section{AUTHOR(S):}

Zu, Guoqing; Shimizu, Taiyo; Kanamori, Kazuyoshi; Zhu, Yang; Maeno, Ayaka; Kaji, Hironori; Shen, Jun; Nakanishi, Kazuki

\section{CITATION:}

Zu, Guoqing ...[et al]. Transparent, Superflexible Doubly Cross-Linked

Polyvinylpolymethylsiloxane Aerogel Superinsulators via Ambient Pressure Drying. ACS Nano 2018, 12(1): 521-532

\section{ISSUE DATE:}

2018

URL:

http://hdl.handle.net/2433/232621

\section{RIGHT:}

This document is the unedited author's version of a Submitted Work that was subsequently accepted for publication in ACS Nano, copyright (C) American Chemical Society after peer review. To access the final edited and published work, see https://doi.org/10.1021/acsnano.7b07117.; この論文は出版社版でありません。引用の際には出版社版をご確認ご利用 ください。; This is not the published version. Please cite only the published version. 


\section{Ultralow-cost, Highly Scalable, Transparent, Superflexible Doubly Crosslinked Polyvinylpolymethylsiloxane Aerogel Superinsulators}

Guoqing Zu, ${ }^{a, c, *}$ Taiyo Shimizu, ${ }^{a}$ Kazuyoshi Kanamori, ${ }^{a, *}$ Yang Zhu, ${ }^{a}$ Ayaka Maeno, ${ }^{b}$ Hironori Kaji, ${ }^{b}$ Jun Shen, ${ }^{c}$ Kazuki Nakanishi ${ }^{a}$

${ }^{a}$ Department of Chemistry, Graduate School of Science, Kyoto University, Kitashirakawa,

Sakyo-ku, Kyoto 606-8502, Japan

bInstitute for Chemical Research, Kyoto University, Gokasho, Uji, Kyoto 611-0011, Japan

'Shanghai Key Laboratory of Special Artificial Microstructure Materials and Technology, Pohl Institute of Solid State Physics, Tongji University, Shanghai 200092, P. R. China
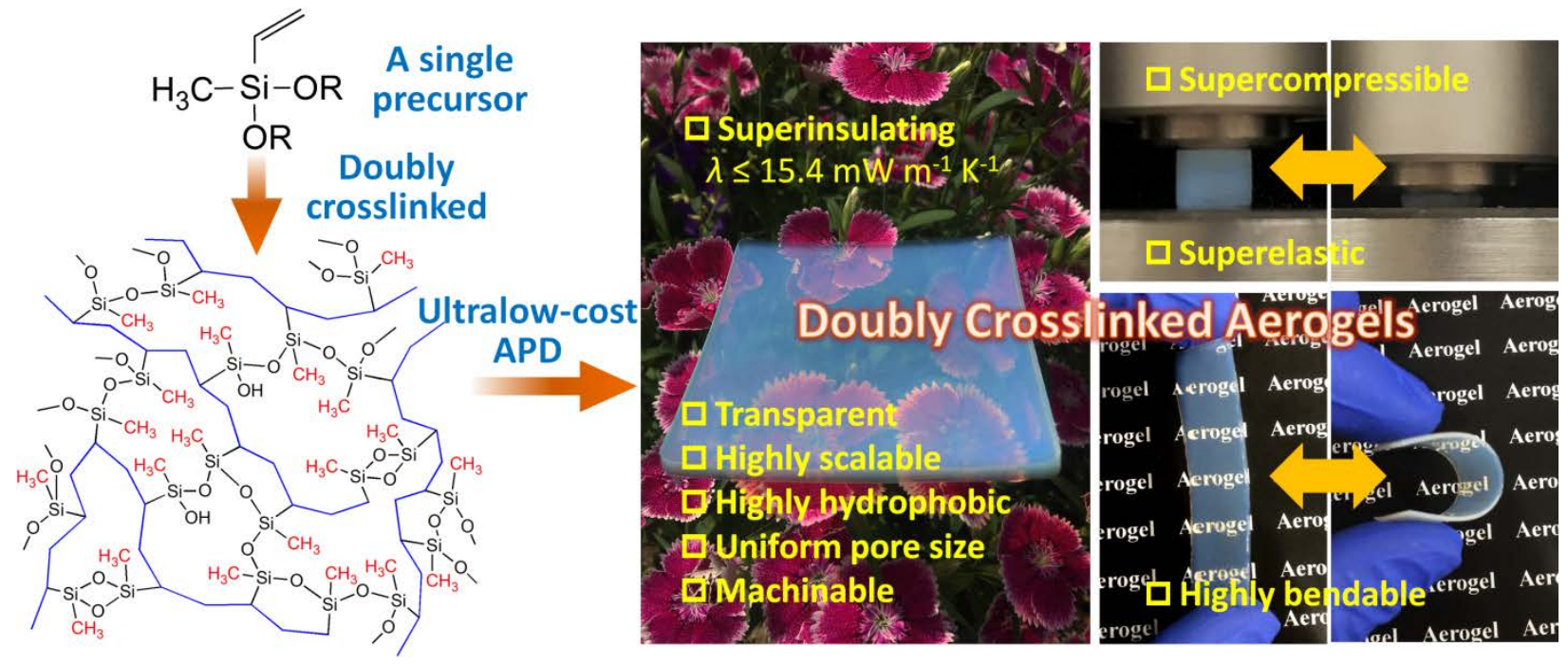


\section{ABSTRACT}

Aerogels have many attractive properties but are usually costly and too friable, which always limit their practical applications. Besides, almost all of the reinforced aerogels sacrifice the transparency or superinsulating properties. Here we report unprecedented superflexible polyvinylpolymethylsiloxane $\left(\left(\mathrm{CH}_{2} \mathrm{CH}\right)_{n}\left(\mathrm{CH}_{3}\right) \mathrm{SiO}_{2 / 2}\right)$ aerogels that are facilely prepared from a single precursor vinylmethyldimethoxysilane or vinylmethyldiethoxysilane without organic crosslinkers. It is based on a consecution of radical polymerization and hydrolytic polycondensation followed by ultralow-cost, highly scalable ambient pressure drying directly from alcohol as a drying medium without any modification and additional solvent exchange. The resulting aerogels and xerogels show a unique, homogeneous, tunable, highly porous, doubly crosslinked nanostructure with the elastic polymethylsiloxane network crosslinked with flexible hydrocarbon chains. An outstanding combination of ultralow cost, high scalability, uniform pore size, high surface area, high transparency, high hydrophobicity, excellent machinability, superflexibility in compression, superflexibility in bending and superinsulating properties has been achieved in a single aerogel and xerogel for the first time. This study represents a significant progress of porous materials and makes the practical applications of transparent flexible aerogel-based superinsulators realistic.

KEYWORDS: polyvinylpolymethylsiloxane aerogel, ultralow cost, transparent, superflexible, superinsulating 
Aerogels are a kind of highly porous materials typically obtained by the sol-gel process combined with a specific drying process. Since they were first prepared in 1931, many types of aerogels such as silica, ${ }^{1,2}$ metal oxide, ${ }^{3,4}$ chalcogenide, ${ }^{5}$ cellulose, ${ }^{6,7}$ polymer, ${ }^{8}$ carbon, ${ }^{9}$ carbon nanotube, ${ }^{10,11}$ and graphene ${ }^{12,13}$ have been developed. Aerogels show a variety of attractive properties such as high porosity, high specific surface area (SSA), low density and low thermal conductivity. ${ }^{3-6}$ Owing to their unique properties, aerogels have drawn great interests in a wide range of applications including thermal insulators, adsorbents, catalyst supports, energy storage, Cherenkov radiators and cosmic dust collectors. ${ }^{3,4,6}$ However, there are two disadvantages that limit the practical applications of aerogels. First, because they are composed mostly of air and their skeletons consist of randomly interconnected nanoparticles, aerogels are usually too friable. Although some aerogels based on nanofibers exhibit compressibility or bendability, 14,15 transparent low-density aerogels combining compressing and bending flexibility are rarely reported. Second, a crucial step for the preparation of aerogels is the drying process, which replaces the liquid solvent within the pores of the gels with air. In order to preserve their friable/deformable porous structure, aerogels are usually prepared via supercritical drying (SCD), which minimizes the contraction of the drying gel but requires high pressure, thus making their preparation energy-consuming and expensive. ${ }^{3}$

To facilitate the drying process, ambient pressure drying (APD) has been developed. ${ }^{16}$ This drying method usually involves multistep solvent exchange with solvent having low surface tension and a modification by silylation of residual silanol groups with an organosilane compound to render the surface hydrophobicity. However, the aerogels prepared via APD usually 
show serious cracking because of the friable structure of the gels, which makes it difficult to obtain low-density monolithic aerogels via APD.

To overcome their friability, significant efforts have been dedicated to enhance the toughness of aerogels by crosslinking with organic compounds. Many robust silica-based composite aerogels have been developed by crosslinking with organic compounds such as isocyanate, epoxide, polyimide and polyacrylate. ${ }^{17-20}$ Unlike native silica aerogels with high visible light transparency, high SSA and low density, however, most of the reinforced aerogels are not transparent in visible light region because of their coarsened microstructure and/or increased inhomogeneity and exhibit lower SSAs and higher density by the incorporation of organic compounds. These changes restrict their applications in thermal superinsulation (especially transparent window insulation), adsorption, catalysis, etc. Besides, this crosslinking method usually adds some tedious steps in the preparation process, which also discourages the practical applications.

Another approach to improve the mechanical properties is to use a trifunctional organoalkoxysilane such as methyltrimethoxysilane/methyltriethoxysilane (MTMS/MTES), ethyltrimethoxysilane (ETMS) or vinyltrimethoxysilane (VTMS) as a single precursor to prepare polyorganosilsesquioxane aerogels. ${ }^{21-23}$ Transparent and elastic polyorganosilsesquioxane aerogels can be obtained by suppressing the macroscopic phase separation of the hydrophobic network via the utilization of an adequate surfactant, but those aerogels do not show bending flexibility. ${ }^{21,22}$ It is reported that incorporating a difunctional organoalkoxysilane in the MTMS system enhances the flexibility but sacrifices the transparency, resulting from the macroscopic 
phase separation of the more hydrophobic network. ${ }^{24}$ Organo-bridged alkoxysilane precursors are also used to improve the mechanical properties of aerogels. ${ }^{25-28}$ Although some of the obtained aerogels based on hexylene-bridged polysilsesquioxane ${ }^{29}$ and ethylene-bridged/ethenylene-bridged polymethylsiloxane ${ }^{27,30}$ are transparent and show bendability due to the organic bridge, their bendability is far from satisfactory. It therefore still remains a great challenge to prepare bendable and elastic aerogels with high transparency.

Radical polymerization of monomers that contain alkene groups is another effective approach to enhance the mechanical properties of aerogels. Optically opaque and mechanically strong silica-based aerogels crosslinked with polystyrene or poly(butyl acrylate) prepared by radical polymerization have been reported. ${ }^{20,31}$ Radical polymerization of vinyl groups in the network of polyvinylsilsesquioxane gels also leads to mechanically reinforced aerogels. ${ }^{23} \mathrm{Up}$ to now, most of the reinforcement by radical polymerization on silica-based aerogels is processed as a post-gelation treatment. It is noteworthy that flexible hybrid wet gels and dense gel films can be obtained by radical polymerization of VTMS, followed by the hydrolytic polycondensation. ${ }^{32,33}$ The polyethylene chains interconnected with siloxane bonds in the network provide flexibility to the hybrid gel. In addition, mechanically strong and flexible organic polymer hydrogels with a double network structure were synthesized via a radical polymerization. ${ }^{34-37}$ The obtained gels contain two interpenetrating soft and sacrificial rigid polymer networks with no chemical crosslinking between two networks. These two examples implicate a probability of further reinforcement of aerogel materials by a doubly crosslinked network, which can be derived by radical polymerization followed by hydrolytic polycondensation from specific 
organoalkoxysilanes with alkene groups for instance. However, to the best of our knowledge, there is no report on the preparation of aerogels via this kind of method.

Here, we report unprecedented superflexible aerogels - polyvinylpolymethylsiloxane (PVPMS, $\left.\left(\mathrm{CH}_{2} \mathrm{CH}\right)_{n}\left(\mathrm{CH}_{3}\right) \mathrm{SiO}_{2 / 2}\right)$ aerogels that have been facilely prepared for the first time by a combination of radical polymerization and hydrolytic polycondensation from a single difunctional precursor with one vinyl group and two alkoxy groups vinylmethyldimethoxysilane (VMDMS) or vinylmethyldiethoxysilane (VMDES), followed by SCD or ultralow-cost APD. Direct hydrolysis and condensation of VMDMS or VMDES do not afford a three-dimensional network structure because it contains only two hydrolysable groups. In our approach (Figure 1a), the precursor is first radically polymerized using di-tert-butyl peroxide (DTBP) as an initiator to afford polyvinylmethyldimethoxysilane (PVMDMS) or polyvinylmethyldiethoxysilane (PVMDES). The conversion and degree of polymerization of PVMDMS or PVMDES are readily controlled by adjusting polymerization time and the initiator concentration. The obtained PVMDMS or PVMDES is then subjected to hydrolysis and condensation with tetramethylammonium hydroxide (TMAOH) as a base catalyst, leading to transparent gels. The aerogels and xerogels are finally obtained after SCD and APD, respectively (Figure 1b). In particular, crack-free aerogel-like xerogels can be obtained by APD directly from alcohol as a drying medium without any modification and additional solvent exchange, which will significantly reduce their cost of production. Both the aerogels and xerogels show a homogeneous, tunable, highly porous nanostructure consisting of flexible hydrocarbon chains and polymethylsiloxanes that are chemically crosslinked each other. Their unique doubly 
crosslinked structure leads to a combination of low density, high SSA, high hydrophobicity, high transparency, excellent machinability, superflexibility in compression and bending and superinsulating properties in a single aerogel and xerogel. These superior properties together with the ultralow-cost APD make their practical applications in transparent flexible superinsulators feasible.

\section{RESULTS AND DISCUSSION}

During the synthesis of PVMDMS or PVMDES polymers, the polymerization temperature is fixed at $120{ }^{\circ} \mathrm{C}$, while the polymerization time is in the range of 6-72 h. Results on the radical polymerization of VMDMS are listed in Table S1 in the Supporting Information. Under the constant DTBP concentration of $1 \mathrm{~mol} \%$, the measured degree of polymerization of PVMDMS with polymerization time of 24, 48 and $72 \mathrm{~h}$ is $40.5,40.5$ and 45.7, respectively. Meanwhile, the corresponding conversion of PVMDMS is 91\%, 95\% and 99\%, respectively, indicating the increase of conversion of PVMDMS with the increase of polymerization time. The resulting PVMDMS with a DTBP concentration of $5 \mathrm{~mol} \%$ and polymerization time of $48 \mathrm{~h}$ exhibits higher degree of polymerization (68.0) and conversion (99\%) compared to those of samples with the DTBP concentration of $1 \mathrm{~mol} \%$. 


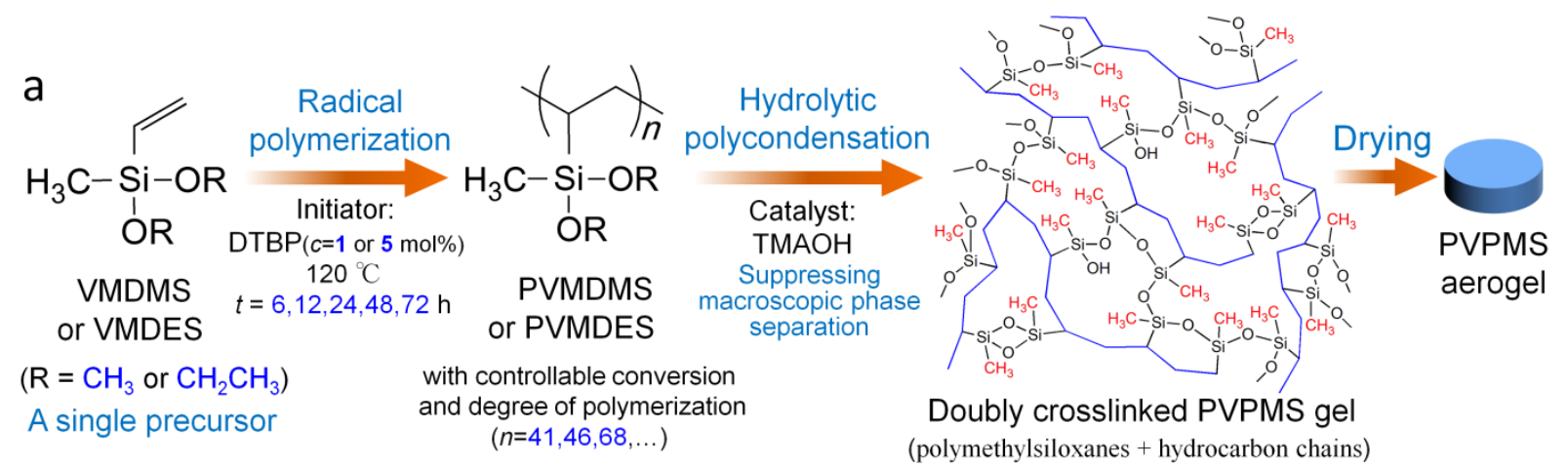

b Comparison of different drying Methods
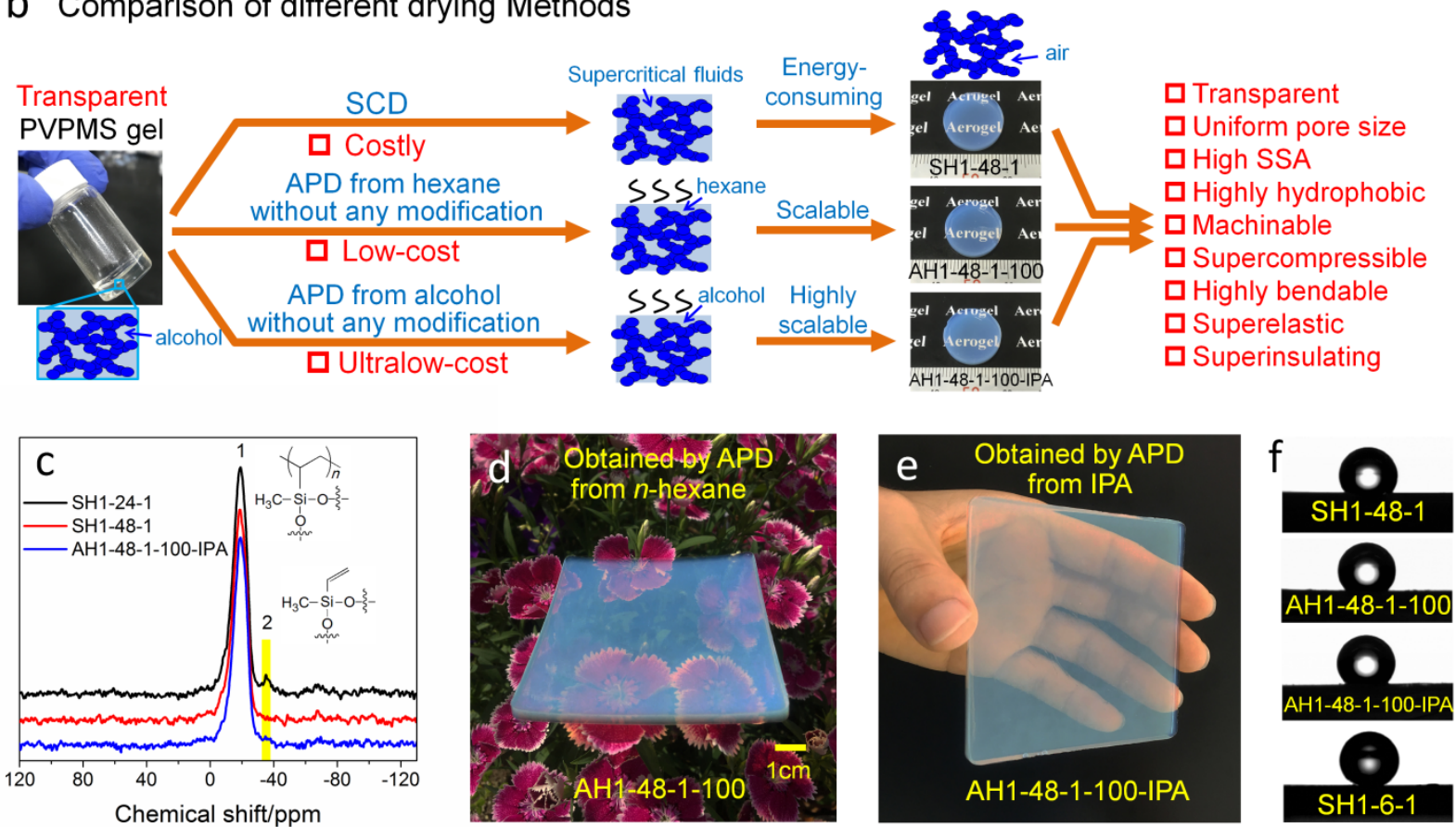

Figure 1. Synthesis route and resulting PVPMS aerogels and xerogels. (a) Schematic of facile synthesis of transparent PVPMS aerogels from a single precursor - VMDMS or VMDES by a combination of radical polymerization and hydrolytic polycondensation followed by SCD or APD. (b) Comparison of different drying methods. (c) Solid-state ${ }^{29}$ Si CP/MAS NMR spectra of typical PVPMS aerogels and xerogels. (d) Photograph of a large APD xerogel panel (AH1-48-1-100, dried from $n$-hexane) with width $\times$ length $\times$ height of $9 \times 9 \times 0.5 \mathrm{~cm}$ on flowers. (e) Photograph of a large APD xerogel panel (AH1-48-1-100-IPA, dried from IPA) with width $\times$ length $\times$ height of $9 \times 9 \times 0.5 \mathrm{~cm}$ in hand. (f) The contact angles of water on SH1-48-1, 
AH1-48-1-100, AH1-48-1-100-IPA and SH1-6-1 are 131 ${ }^{\circ}, 133^{\circ}, 133^{\circ}$ and $154^{\circ}$, respectively.

Table 1 and Table S2 detail the starting compositions and typical physical properties of the resulting PVPMS aerogels and xerogels. The aerogel samples obtained by SCD are given the prefix SH, while those by APD are denoted as AH. The number 1 and 5 after the character indicates the aerogels with the DTBP concentration of $1 \%$ and $5 \%$, respectively. The following number specifies the polymerization time of VMDMS. The third number 1, 2 and 3 indicates the aerogels with the molar ratio of $\mathrm{BzOH}$ to VMDMS of 4.3, 5.0 and 5.7, respectively. Some of the sample names are followed by the number 100 , indicating the rigorous aging at $100{ }^{\circ} \mathrm{C}$. The sample names without number 100 indicate the aging temperature of $80^{\circ} \mathrm{C}$. The following character "IPA" indicates APD from 2-propanol (IPA) as a drying medium, while those without "IPA" were dried by SCD or APD from $n$-hexane. The last character "E" indicates the aerogels obtained from VMDES, while those without "E” were obtained from VMDMS.

The polymerization time shows a significant influence on the microstructures and properties of the resulting PVPMS aerogels. As presented in the FTIR spectra (Figure S1), the residual vinyl groups (=C-H stretching at $3056 \mathrm{~cm}^{-1}, \mathrm{C}=\mathrm{C}$ stretching at $1600 \mathrm{~cm}^{-1},=\mathrm{CH}_{2}$ scissor at $1406 \mathrm{~cm}^{-1}$ overlapped with $-\mathrm{C}-\mathrm{H}$ deformation, and $=\mathrm{C}-\mathrm{H}$ bending at $\left.532 \mathrm{~cm}^{-1}\right)^{38}$ in the aerogel gradually decrease with the increase of polymerization time. For the sample with polymerization time of $24 \mathrm{~h}$ (SH1-24-1), the absorption bands corresponding to vinyl groups are already too weak to be identified in FTIR spectra, while for the sample with polymerization time of $48 \mathrm{~h}$ (SH1-48-1), there is no vinyl group absorption observed. Besides, by the solid-state ${ }^{29} \mathrm{Si}$ cross-polarization 
magic angle spinning (CP/MAS) NMR spectra (Figure1c), a peak at around -35ppm corresponding to the silicon with vinyl groups $\left(\mathrm{CH}_{2}=\mathrm{CH}\left(\mathrm{CH}_{3}\right) \mathrm{SiO}_{2 / 2}\right)$ is detected on $\mathrm{SH} 1-24-1$, while it is not observed on SH1-48-1. This is consistent with the results of the different conversion values of PVMDMS for these two samples.

Table 1. Compositions and typical physical properties of the PVPMS aerogels and xerogels. ${ }^{\text {a) }}$

\begin{tabular}{|c|c|c|c|c|c|c|c|c|c|c|c|}
\hline sample & $\begin{array}{l}\text { DTBP } \\
/ \mathrm{mol} \%\end{array}$ & $\begin{array}{l}t_{\mathbf{p}}^{\mathbf{b})} \\
/ \mathbf{h}\end{array}$ & $\begin{array}{c}\mathrm{BzOH} / \mathrm{Si} \\
/ \mathrm{mol} \mathrm{mol}^{-1}\end{array}$ & $\begin{array}{c}\mathrm{H}_{2} \mathrm{O} / \mathrm{Si} \\
/ \mathrm{mol} \mathrm{mol}^{-1}\end{array}$ & $\begin{array}{c}\text { TMAOH/Si } \\
/ \mathrm{mol} \mathrm{mol}^{-1}\end{array}$ & $\begin{array}{c}\rho^{\mathrm{c})} \\
/ \mathrm{g} \mathrm{cm}^{-3}\end{array}$ & $\begin{array}{l}S_{\mathrm{BET}}{ }^{\mathrm{d})} \\
/ \mathbf{m}^{2} \mathbf{g}^{-1}\end{array}$ & $\begin{array}{c}d^{\mathrm{e})} \\
/ \mathbf{n m}\end{array}$ & $\begin{array}{c}\left.V_{p} \mathbf{f}\right) \\
/ \mathbf{c m}^{3} \mathbf{g}^{-1}\end{array}$ & $\begin{array}{l}T^{\mathrm{g})} \\
/ \%\end{array}$ & $\begin{array}{c}\lambda^{\mathbf{h})} \\
/ \mathbf{m W} \mathbf{m}^{-1} \mathbf{K}^{-1}\end{array}$ \\
\hline SH1-48-1 & 1 & 48 & 4.3 & 2.0 & 0.030 & 0.22 & 950 & 32.3 & 3.64 & 82.4 & 15.3 \\
\hline AH1-48-1 & 1 & 48 & 4.3 & 2.0 & 0.030 & 0.31 & 912 & 24.4 & 2.38 & 90.2 & - \\
\hline AH1-48-1-100 & 1 & 48 & 4.3 & 2.0 & 0.030 & 0.21 & 906 & 43.6 & 3.95 & 80.6 & 15.4 \\
\hline AH1-48-1-100-IPA & 1 & 48 & 4.3 & 2.0 & 0.030 & 0.21 & 903 & 43.6 & 3.91 & 80.2 & 15.4 \\
\hline AH5-48-1-100-IPA & 5 & 48 & 4.3 & 2.0 & 0.030 & 0.22 & 916 & 32.3 & 3.66 & 84.3 & 15.2 \\
\hline SH1-48-2 & 1 & 48 & 5.0 & 2.0 & 0.045 & 0.19 & 919 & 43.6 & 4.47 & 76.4 & 15.2 \\
\hline AH1-48-2-100 & 1 & 48 & 5.0 & 2.0 & 0.045 & 0.18 & 908 & 50.4 & 4.70 & 75.5 & 15.2 \\
\hline AH1-48-2-100-IPA & 1 & 48 & 5.0 & 2.0 & 0.045 & 0.18 & 905 & 50.4 & 4.64 & 75.3 & - \\
\hline AH5-48-2-100-IPA & 5 & 48 & 5.0 & 2.0 & 0.045 & 0.19 & 912 & 43.6 & 4.55 & 78.1 & 15.1 \\
\hline SH1-48-3 & 1 & 48 & 5.7 & 2.0 & 0.067 & 0.16 & 937 & 58.1 & 5.37 & 65.7 & 15.0 \\
\hline
\end{tabular}

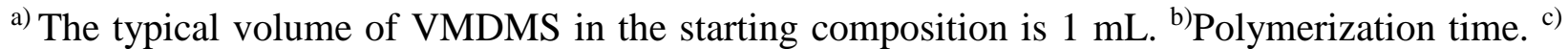
Bulk density. d) Brunauer-Emmett-Teller (BET) SSA obtained from nitrogen adsorption measurement. e) Mean pore diameter obtained from nitrogen adsorption branch via

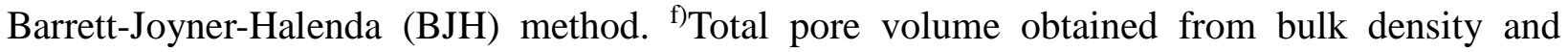
skeletal density. g) Visible-light transmittance at $550 \mathrm{~nm}$ for 2-mm thick aerogel. h) Thermal conductivity at room temperature and ambient pressure.

The unpolymerized VMDMS in the precursor solution tends to form cyclic and chain-like species during the hydrolytic polycondensation process, which results in inhomogeneous 
gelation of the sol. In this case, macroscopic phase separation between the hydrophobic condensates and polar solvent occurs, resulting in precipitations or monolithic gels with coarsened domains and low transparency ${ }^{21}$. This tendency is supposed to be lower in PVMDMS compared to that of VMDMS, because the resulting PVMDMS molecules possess a large number of hydrolysable alkoxy groups, giving higher crosslinking density and promoting homogenous gelation. As a result, under the constant DTBP concentration of $1 \mathrm{~mol} \%$, the aerogels with polymerization time of $6 \mathrm{~h}$ and $12 \mathrm{~h}$ are opaque and translucent, respectively (Figure S2a), whereas those with polymerization time of 24, 48, and $72 \mathrm{~h}$ are transparent with bluish appearance caused by the Rayleigh scattering of short-wavelength light by their ultrafine particles (Figure1b and Figure S2a). The 2-mm thick PVPMS aerogels with bulk density in the range $0.21-0.31 \mathrm{~g} \mathrm{~cm}^{-3}$ and polymerization time of $48 \mathrm{~h}$ and $72 \mathrm{~h}$ show a high visible-light transmittance of 80-90\% (Table 1 and Table S2).

The SSAs of the aerogels increase and the pore and particle size decrease with the increase of polymerization time in the range 6-72h (Table 1, Table S2, Figure S2b-d, and Figure S3). The PVPMS aerogels with polymerization time of 24,48 and $72 \mathrm{~h}$ exhibit a high SSA of $>900 \mathrm{~m}^{2} \mathrm{~g}^{-1}$, apparently higher than those of aerogels with shorter polymerization time (248 and $701 \mathrm{~m}^{2} \mathrm{~g}^{-1}$ for SH1-6-1 and SH1-12-1, respectively). Meanwhile, the mean pore diameter decreases from $>100 \mathrm{~nm}$ for SH1-6-1 to $58.1 \mathrm{~nm}$ for SH1-12-1 and then $28.1 \mathrm{~nm}$ for SH1-72-1. The aerogel with polymerization time of $6 \mathrm{~h}$ (SH1-6-1) exhibits large pores and aggregated particles (Figure S2b), which results from the phase separation mainly due to the less crosslinked hydrophobic condensates from less polymerized species. The longer polymerization time leads to 
a more homogenous structure and thinner skeletons of the resulting aerogels. The microstructure and properties of the PVPMS aerogels can be regulated by varying the polymerization time of VMDMS (Figure S2e).

Due to the methyl group and vinyl group bonded to silicon of the precursor VMDMS, there are abundant methyl groups and aliphatic hydrocarbon chains and only a small amount of $-\mathrm{OH}$ groups in the network of the resulting PVPMS aerogels, which is evidenced by the FTIR spectra (Figure S1) and solid-state ${ }^{29} \mathrm{Si}$ CP/MAS NMR spectra (Figure 1c). As shown in FTIR spectra, the absorption bands corresponding to $\mathrm{C}-\mathrm{H}$ and $\mathrm{Si}-\mathrm{C}$ bonds indicate the presence of methyl groups and/or the aliphatic hydrocarbon chains of the PVPMS aerogels. ${ }^{24,39,40}$ As presented in NMR spectra, an intense peak located at $-19 \mathrm{ppm}$, which corresponds to $\mathrm{D}^{2}$ $\left(\left(\mathrm{CH}_{2} \mathrm{CH}\right)_{n}\left(\mathrm{CH}_{3}\right) \underline{\mathrm{SiO}}_{2 / 2}\right)$ species, ${ }^{27}$ shows the presence of methyl groups, aliphatic hydrocarbon chains and polysiloxanes in the PVPMS aerogels. Due to their unique structures with abundant hydrophobic groups and only a small amount of $-\mathrm{OH}$ groups, the PVPMS aerogels exhibit high hydrophobicity. Both the aerogels and xerogels can float on top of water for at least 6 months (Figure S4). The contact angles of water on all the samples are found to be larger than $130^{\circ}$ (Figure 1f), of which the samples with shorter polymerization time show better hydrophobicity because of the rougher surfaces, as confirmed by the FESEM images (Figure S2b-d). For example, SH1-6-1 shows superhydrophobicity with a contact angle as high as $154^{\circ}$. 

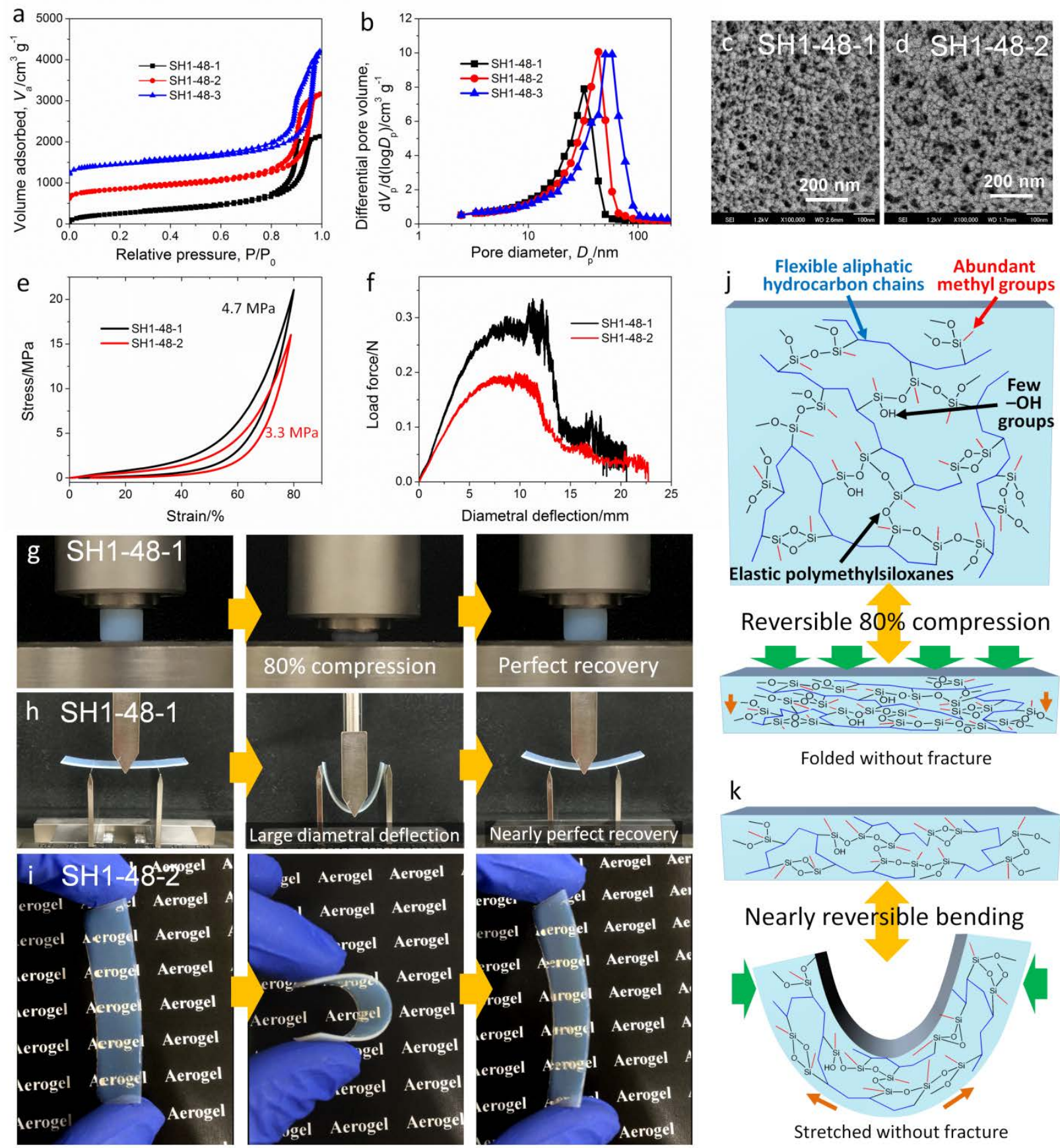

Reversible $80 \%$ compression
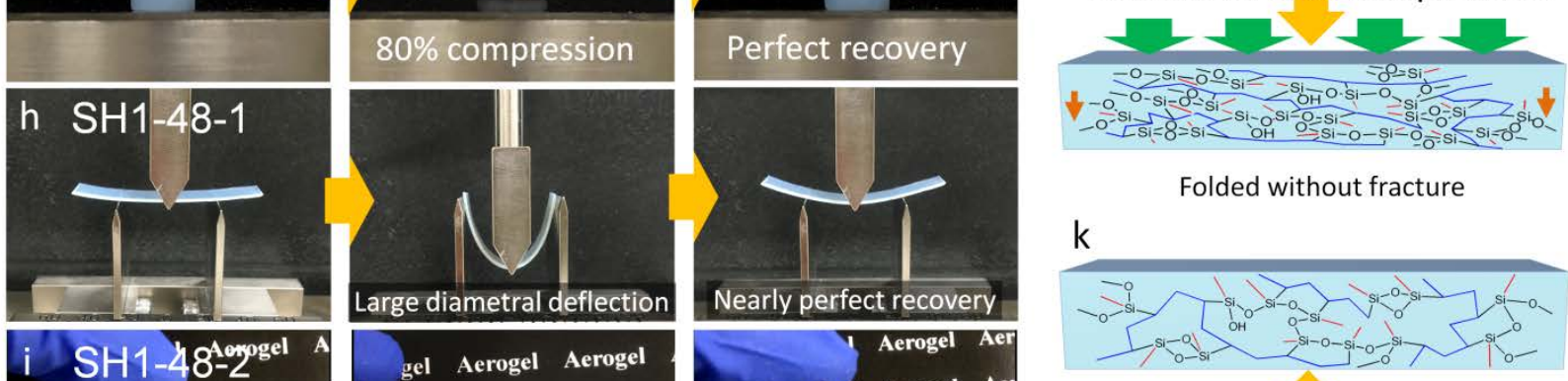

Nearly reversible bending

Figure 2. Porous structure and mechanical properties of PVPMS aerogels. (a) $\mathrm{N}_{2}$ adsorption/desorption isotherms and (b) pore size distributions of typical PVPMS aerogels obtained by SCD. The y-axis shift in each isotherm is $600 \mathrm{~cm}^{3} \mathrm{~g}^{-1}$. FESEM images of typical PVPMS aerogels by SCD: (c) SH1-48-1 and (d) SH1-48-2. (e) Stress-strain curves of uniaxial 
compression-decompression tests on typical PVPMS aerogels. The values in the figure are the obtained Young's moduli. (f) Stress-strain curves of three-point bending tests on typical PVPMS aerogels. (g) Photographs of a uniaxial compression-decompression test on SH1-48-1. (h) Photographs of a three-point bending test on SH1-48-1. The thickness, width and length of the sample are $1 \mathrm{~mm}, 10 \mathrm{~mm}$ and $50 \mathrm{~mm}$, respectively and the fixture span is $25 \mathrm{~mm}$. (i) Photographs of SH1-48-2 during a hand bending test. It shows a large bending deformation without fracture when it is bent by hand, then recovers nearly its original shape after it is released. Schematic of molecular-scaled structure variations during (j) compression and (k) bending tests.

In order to obtain transparent PVPMS aerogels, it is crucial to optimize the catalyst parameters such as the type and concentration to control hydrolysis and polycondensation of PVMDMS. Here, a strong base TMAOH is used as the hydrolysis and polycondensation catalyst to promote gelation. As reported, stronger bases contribute to more accelerated gelation prior to macroscopic phase separation due to higher $\mathrm{pH}$ of the solution, which increases the homogeneity of the porous structure and transparency of the resulting aerogels. ${ }^{21}$ It is clearly observed from Figure 2a that the resulting PVPMS aerogels exhibit type IV isotherms with distinct capillary condensation steps at the relative pressure of $0.5-1.0$, demonstrating the mesoporous three-dimensional network structure of the aerogels. Figure 2b-d further confirms that they show a randomly interconnected homogenous porous structure that is composed of aggregated nanoparticles. The pore and particle sizes of SH1-48-1 are mainly 10-40 and 10-35 nm, respectively. Besides, 
SH1-48-1 shows a high SSA (950 $\left.\mathrm{m}^{2} \mathrm{~g}^{-1}\right)$ and high transparency (82.4\% transmittance for 2-mm thick aerogel).

The precursor concentration also significantly affects the structure of the PVPMS aerogels. According to a report, ${ }^{21}$ trifunctional alkoxysilanes show a higher tendency for inhomogeneous gelling when the precursor concentration is low. Similarly, PVPMS aerogels with the lower precursor concentration are supposed to possess the more inhomogeneous structure. It is found that the optimized aerogels with a lower precursor concentration (SH1-48-2 and SH1-48-3) show larger particle size and wider pore size distribution compared with the samples with a higher precursor concentration (Figure 2b-d). Since a larger pore size results in stronger visible light scattering, the PVPMS aerogels with a lower precursor concentration show relatively lower transparency. The visible-light transmittance decreases from $82.4 \%$ for SH1-48-1 to $76.4 \%$ for SH1-48-2 and then $65.7 \%$ for SH1-48-3. Evidently, the structure and properties of the PVPMS aerogels can also be regulated by varying the precursor concentration.

Benefiting from their unique molecular- and nano-scaled structures, the PVPMS aerogels exhibit excellent mechanical properties. As presented in the stress-strain curves and photographs of uniaxial compression-decompression tests and the three-point bending tests (Figure 2e-h), the optimized aerogels SH1-48-1 and SH1-48-2 combine high compressive flexibility (strength plus recovery) and high bending flexibility. They are compressed with $80 \%$ strain without fracture and then perfectly recover their original shape after the force is removed. Meanwhile, they are able to recover nearly their original shape from a large diametral deflection of around 18-25 mm in the three-point bending tests with a fixture span of $25 \mathrm{~mm}$. The hand bending tests of 
SH1-48-2 (Figure 2i and Movie S1) clearly demonstrate the high bending flexibility of PVPMS aerogels. The Young's moduli of SH1-48-1 and SH1-48-2 calculated from the stress-strain curves of uniaxial compression-decompression tests is 4.7 and $3.3 \mathrm{MPa}$, respectively, which is comparable to that for traditional silica ${ }^{41}$ and polymethylsilsesquioxane (PMSQ) aerogels. ${ }^{21}$ The Poisson's ratio of SH1-48-1 is around 0.1, which is similar to that of PMSQ aerogels (0.12). ${ }^{21}$

There are three main reasons contribute to the excellent mechanical properties of the PVPMS aerogels. First, the elastic polymethylsiloxane network in the skeletons contains abundant methyl groups, which facilitates their recovery from compression and bending. Second, the polymethylsiloxane network is crosslinked with flexible aliphatic hydrocarbon chains, which leads to the high deformability without fracture and recovery of the skeletons on compression and bending. Third, there are only a small amount of $-\mathrm{OH}$ groups in the skeletons, which reduce the irreversible shrinkage. The molecular-scaled structure variations during compression and bending tests are schematically represented in Figure 2j,k. 

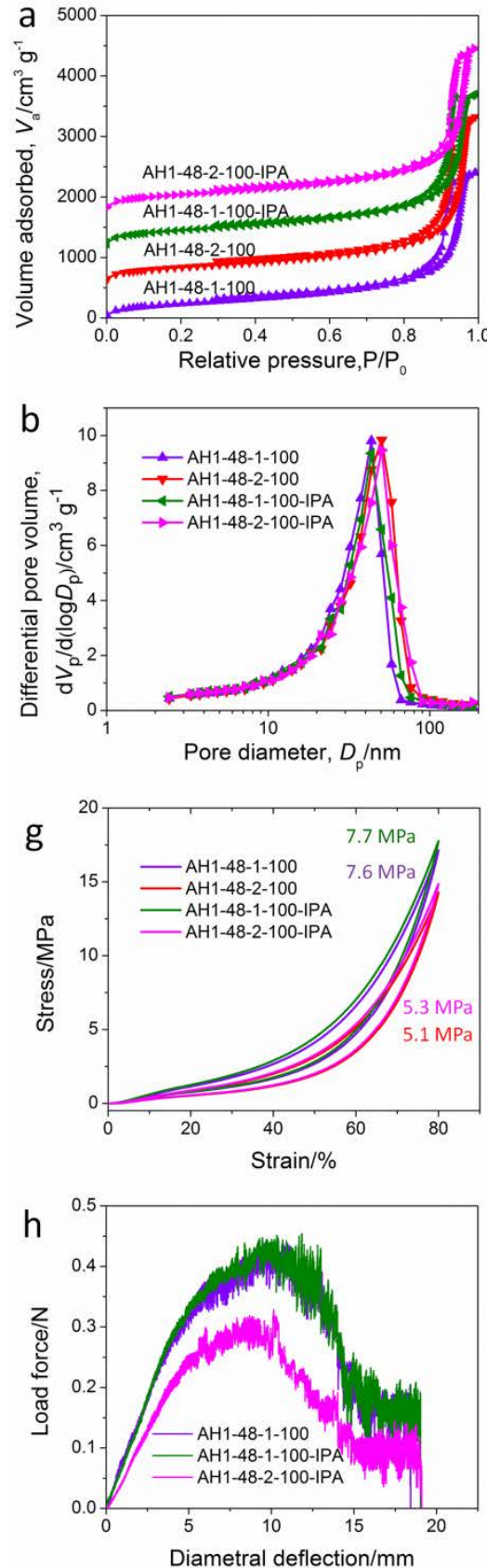
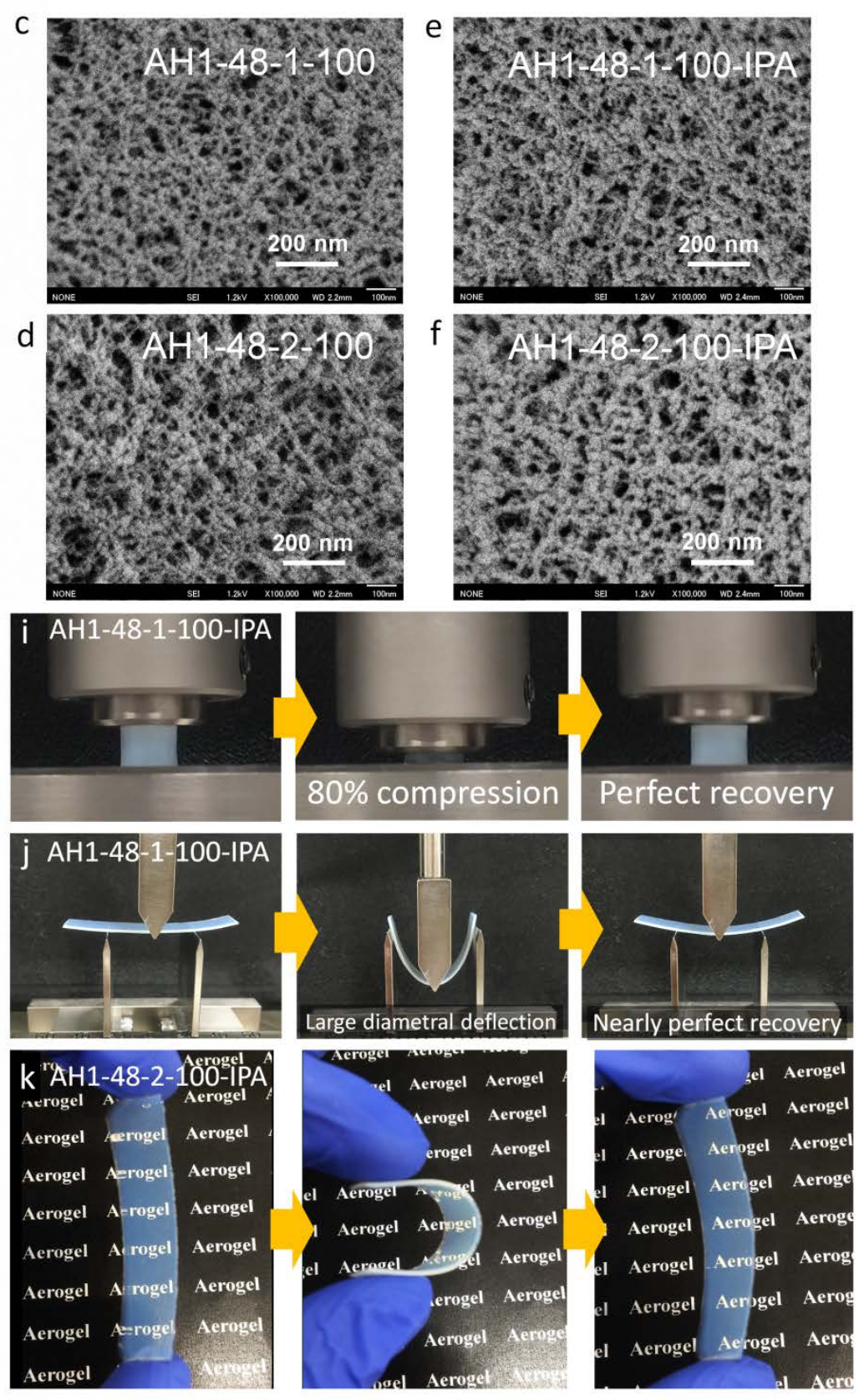

Figure 3. Porous structure and mechanical properties of PVPMS xerogels. (a) $\mathrm{N}_{2}$ adsorption/desorption isotherms and (b) BJH pore size distributions of typical PVPMS xerogels obtained by APD. FESEM images of typical PVPMS xerogels: (c) AH1-48-1-100, (d) AH1-48-2-100, (e) AH1-48-1-100-IPA and (f) AH1-48-2-100-IPA. (g) Stress-strain curves of uniaxial compression-decompression tests on typical xerogels. The values in the figure are the 
obtained Young's moduli. (h) Stress-strain curves of three-point bending tests on the PVPMS xerogels (AH1-48-1-100, AH1-48-1-100-IPA and AH1-48-2-100-IPA). (i) Photographs of a uniaxial compression-decompression test on AH1-48-1-100-IPA. (j) Photographs of a three-point bending test on AH1-48-1-100-IPA. The size of the sample is the same as those of corresponding aerogels. (k) A hand bending test on AH1-48-2-100-IPA showing high bending flexibility.

Due to their unique flexibility, optimized PVPMS wet gels can be successfully dried by APD to afford transparent superflexible PVPMS xerogels with comparable properties to those of the corresponding aerogels. The type IV isotherms with distinct capillary condensation (Figure 3a and Figure S5a) and the narrow pore size distribution (Figure 3b and Figure S5b), together with the FESEM images (Figure 3c-f and Figure S5c), demonstrate that the PVPMS xerogels also show a homogenous mesoporous three-dimensional interconnected network structure. However, the xerogel AH1-48-1 aged at $80{ }^{\circ} \mathrm{C}$ without modifications show higher density $\left(0.31 \mathrm{~g} \mathrm{~cm}^{-3}\right)$ compared with the corresponding aerogel. This variation results from partially irreversible shrinkage caused by the condensation of a small amount of neighboring $-\mathrm{OH}$ groups on the skeletons during the temporal contraction in drying. Due to the smaller pore size (Figure S5b), AH1-48-1 exhibits a higher visible-light transmittance (90.2\%) compared to those of the corresponding aerogels.

It is found that transparent monolithic aerogel-like xerogels with negligible shrinkage (AH1-48-1-100 and AH1-48-2-100) can be obtained by simply increasing the aging temperature to $100{ }^{\circ} \mathrm{C}$ without any modification followed by APD from $n$-hexane. Upon APD, AH1-48-1-100 
and AH1-48-2-100 wet gels undergo a large temporal linear shrinkage of around 21\% due to the capillary force exerted on the entire gel skeletons and then spring back to nearly their original size, resulting from their elastic molecular structure with abundant methyl groups and aliphatic hydrocarbon chains and few -OH groups. The lower irreversible shrinkage during APD compared to those of xerogels aged at $80{ }^{\circ} \mathrm{C}$ probably results from the lower - $\mathrm{OH}$ concentration on the skeletons, achieved by more intense condensation reaction during aging at the higher temperature. It is noteworthy that crack-free large xerogel (AH1-48-1-100) panels (Figure 1d) are obtained via their simple scale-up. Since they are obtained by APD without any modification that consumes surface modifiers, additional solvent and processing time, the production cost for the xerogels is greatly reduced, which will significantly contributes to their practical applications. 
a

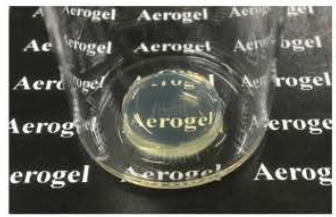

PVPMS alcogel with IPA as the solvent without any modification
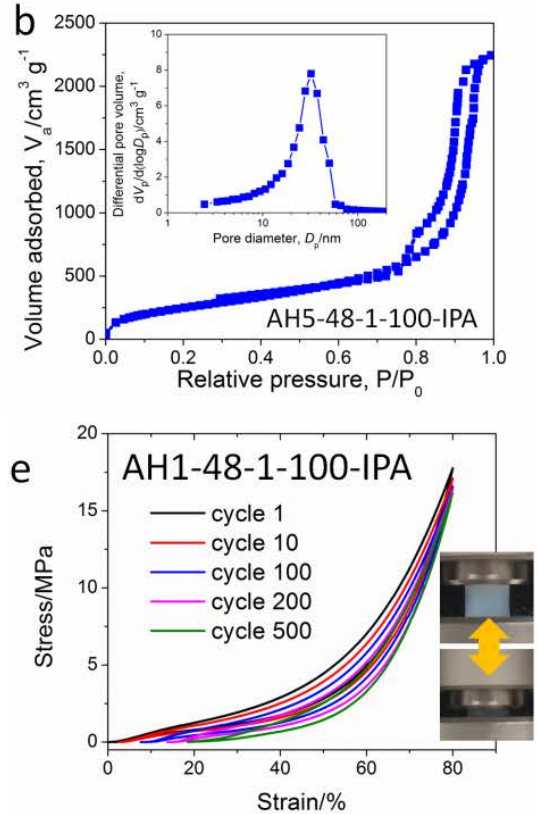

h

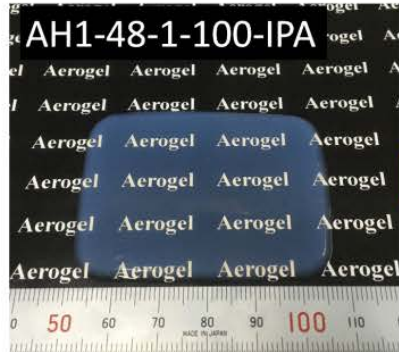

c
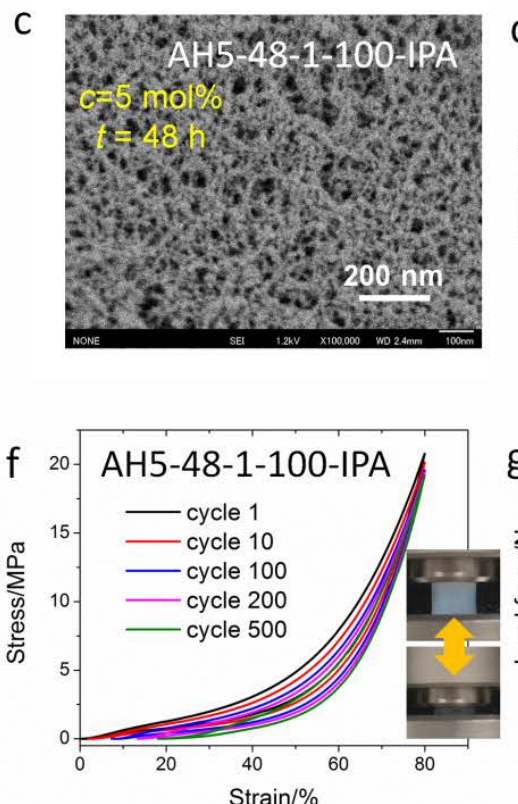

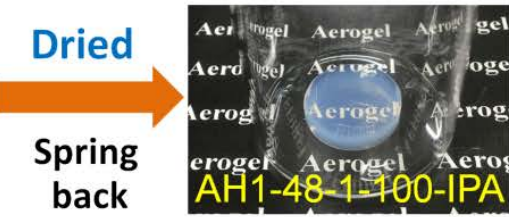

PVPMS

aerogel-like xerogel
Large shrinkage

without fracture
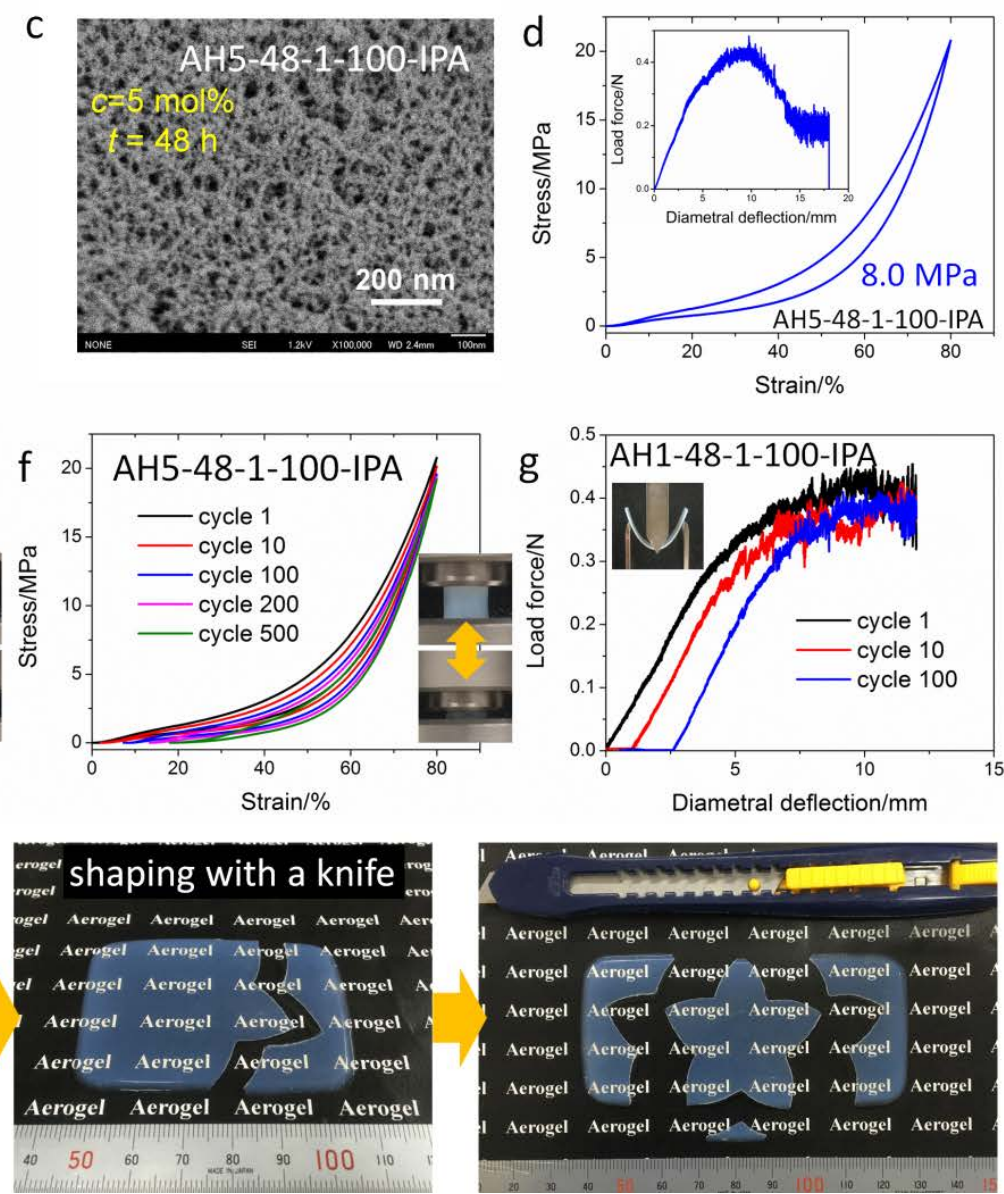

Figure 4. Drying process, structure, compressive and bending cycle performances and machinability. (a) Photographs of AH1-48-1-100-IPA during ultralow-cost APD from IPA as the drying medium without any modification. (b) $\mathrm{N}_{2}$ adsorption/desorption isotherm and (c) FESEM image of AH5-48-1-100-IPA. The inset is its BJH pore size distribution. (d) Stress-strain curves of a uniaxial compression-decompression test on AH5-48-1-100-IPA. The inset is its stress-strain curve of a three-point bending test. Stress-strain curves of 500 cycles of a uniaxial 
compression-decompression test with $80 \%$ strain on (e) AH1-48-1-100-IPA and (f) AH5-48-1-100-IPA. (g) Stress-strain curves of 100 cycles of a three-point bending test with a large diametral deflection of $12 \mathrm{~mm}$ on AH1-48-1-100-IPA. (h) Excellent machinability of the PVPMS xerogel AH1-48-1-100-IPA shown by shaping with a knife.

Transparent monolithic low-density PVPMS xerogels (AH1-48-1-100-IPA and AH1-48-2-100-IPA) can also be obtained from the wet gels aged at $100{ }^{\circ} \mathrm{C}$ via APD directly from IPA as a drying medium without any modification and additional solvent exchange. Because of the larger surface tension of IPA compared to that of $n$-hexane, larger capillary force is exerted on the entire gel skeletons, which results in a larger temporal linear shrinkage (around 30\%) during APD. As shown in Figure 4a, however, they still spring back to nearly their original size without fracture during APD, successfully affording transparent aerogel-like xerogels. Moreover, crack-free large xerogel (AH1-48-1-100-IPA) panels with width $\times$ length $\times$ height of $90 \times 90 \times 5 \mathrm{~mm}$ (Figure 1e) can also be obtained via a simple scale-up. This drying method consumes minimal solvent and energy and no modifiers, which leads to a further reduction of their production cost. The PVPMS xerogels obtained via this ultralow-cost drying method show significant advantages in practical applications over other aerogels obtained via common methods such as SCD, traditional APD with hexamethyldisilazane (HMDS) or trimethylchlorosilane (TMCS) modification, vacuum drying and freeze drying.

Transparent PVPMS xerogels can also be obtained from VMDMS with the DTBP concentration of $5 \mathrm{~mol} \%$ via the same drying method. Since the degree of polymerization and 
conversion of PVMDMS are higher than those of PVMDMS with the DTBP concentration of 1 mol\%, the resulting PVMDMS molecule possesses more hydrolysable groups, leading to higher crosslinking density and more homogenous gelation (Figure S2e). Consequently, as shown in Table 1 and Figure 4b,c the xerogel AH5-48-1-100-IPA possesses smaller pores (mainly 10-45 $\mathrm{nm}$ ) and particles (mainly 10-40 nm) and higher transparency (84.3\%).

The PVPMS xerogels such as AH1-48-1-100-IPA are thermally stable up to around $200{ }^{\circ} \mathrm{C}$, above which the aliphatic hydrocarbon chains and methyl groups are gradually oxidized (Figure S6). As shown in FTIR (Figure S1) and solid-state ${ }^{29}$ Si CP/MAS NMR spectra (Figure 1c), the PVPMS xerogel AH1-48-1-100-IPA exhibits a comparable molecular-scaled structure to those of SCD aerogel. Furthermore, other properties of the xerogels AH1-48-1-100, AH1-48-1-100-IPA and AH1-48-2-100-IPA such as the contact angle of water $\left(133^{\circ}, 133^{\circ}\right.$ and $135^{\circ}$, respectively) (Figure 1f), visible-light transmittance $(80.6 \%, 80.2 \%$ and $75.3 \%$, respectively) (Table 1), pore size (mainly 10-55, 10-55 and 15-70 nm, respectively) (Figure 3b), particle size (mainly 15-55, 15-55 and 15-70 $\mathrm{nm}$, respectively) (Figure 3c-f), pore volume (3.95, 3.91 and $4.64 \mathrm{~cm}^{3} \mathrm{~g}^{-1}$, respectively) (Table 1) and the Young's moduli (7.6, 7.7 and 5.3 MPa, respectively) (Figure 3g) are also similar to those of the corresponding aerogels.

More importantly, the optimized APD xerogels also exhibit excellent mechanical properties that are comparable to those of SH1-48-1 and SH1-48-2 aerogels. As confirmed in Figure 3g-k and Figure 4d, AH1-48-1-100, AH1-48-1-100-IPA, AH1-48-2-100-IPA and AH5-48-1-100-IPA combine high compressive and bending flexibility. They are compressed with $80 \%$ strain without fracture and then completely and rapidly spring back to their original size after the force is 
removed. Movie S2 also shows the high compressive flexibility of AH1-48-1-100-IPA. After compression-decompression for 100 cycles, they still recover nearly their original size (Figure 4e,f and Figure S7a), and their stress-strain curve, SSA, pore size and three-dimensional interconnected nanostructure remain virtually unchanged (Figure S7b-d). It is noteworthy that the PVPMS xerogels such as AH1-48-1-100-IPA and AH5-48-1-100-IPA spring back to approximately $82 \%$ of their original size immediately after the compression-decompression with $80 \%$ strain for 500 cycles (Figure 4e,f) and continue to spring back to around $88 \%$ after $1 \mathrm{~h}$ at room temperature. Furthermore, they recover nearly their original size ( 94\%) with their porous structure nearly unchanged (Figure S8) after heat treatment at $120^{\circ} \mathrm{C}$ for $2 \mathrm{~h}$. This interesting spring-back phenomenon is probably because the flexible skeleton is folded inward toward the pores during compression, remains folded in the pores just after the force is removed, gradually and partially springs back at room temperature and then continues to spring back due to the repulsion and relaxation of the methyl- and aliphatic hydrocarbon chain-rich network during heat treatment.

They also recover nearly their original shape from a large diametral deflection of around 17-20 $\mathrm{mm}$ in the three-point bending tests. The hand bending test of AH1-48-2-100-IPA (Figure 3k and Movie S3) clearly presents the high bending flexibility of PVPMS xerogels. No fracture is observed after bending with a large diametral deflection of $12 \mathrm{~mm}$ for 100 cycles (Figure 4g), indicating an excellent bending cycle performance. In addition, as presented in Figure S9, the PVPMS xerogel AH1-48-2-100-IPA-E obtained from VMDES exhibits the similar structure and bending flexibility to those of AH1-48-2-100-IPA obtained from VMDMS. Furthermore, the 
desired shape can be obtained simply by cutting with a knife, indicating excellent machinability of the PVPMS xerogels (Figure 4h and Figure S10). These unique mechanical properties of PVPMS aerogels and xerogels are superior to those of traditional aerogels. Their superelasticity against compression and bending with large strain is not observed with silica, ${ }^{3,41}$ metal oxide, ${ }^{42}$ and cellulose aerogels ${ }^{6}$ and conventional polymer, ${ }^{43,44}$ carbon $^{9}$ and silica-based organic-inorganic hybrid aerogels. ${ }^{17,20}$ Their bending flexibility is also better than that of PMSQ, 21,22 organo-bridged polysilsesquioxane and polymethylsiloxane aerogels. ${ }^{25,29,30}$

The unique nanostructures of PVPMS aerogels not only lead to excellent mechanical properties but also result in outstanding thermal insulation properties. The total thermal conductivity $\left(\lambda_{\text {total }}\right)$ of porous materials mainly consists of three components: solid $\left(\lambda_{\mathrm{s}}\right)$ and gas $\left(\lambda_{\mathrm{g}}\right)$ conductivities and radiation $\left(\lambda_{\mathrm{r}}\right):^{22}$

$$
\lambda_{\text {total }}=\lambda_{\mathrm{s}}+\lambda_{\mathrm{g}}+\lambda_{\mathrm{r}}(1)
$$

Since the optimized PVPMS aerogels exhibit low density (0.18-0.22 $\mathrm{g} \mathrm{cm}^{-3}$ ) and a homogenous three-dimensional network structure consisting of aggregated nanoparticles, their solid thermal conductivity is supposed to be low. The gas thermal conductivity is given by the following equation: ${ }^{22,45}$

$$
\lambda_{\mathrm{g}}=\phi \frac{\lambda_{\mathrm{g}}^{0}}{1+2 \beta l_{\mathrm{mfp}} p_{0} /[p D]}
$$

where $\phi$ is the porosity, $\lambda_{\mathrm{g}}^{0}$ is the thermal conductivity of non-convective free gas molecules, $\beta$ is a constant specific to the gas in the pores, $l_{\mathrm{mfp}}$ is the mean free path of a gas molecule, $p_{0}$ is the reference gas pressure, $p$ is the gas pressure, $D$ is the average pore size in the porous material. 
Because the optimized PVPMS aerogels and xerogels possess a small pore size mainly in the range of 10-60 nm, which is smaller than the mean free path $(\sim 70 \mathrm{~nm})$ of main molecules in the atmosphere, their gas thermal conductivity is suppressed. The contribution of radiation to the total thermal conductivity is small at room temperature. The optimized PVPMS aerogels and xerogels are therefore supposed to exhibit low total thermal conductivity.

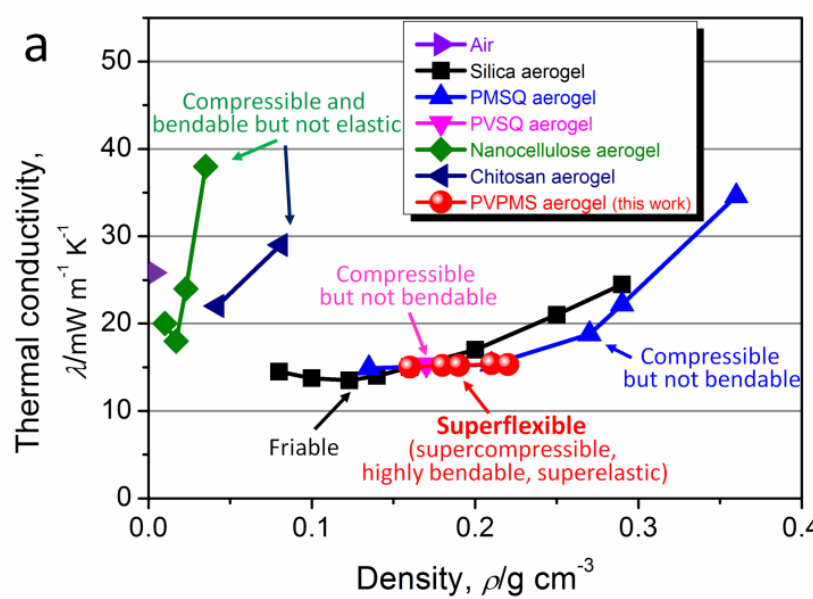

C

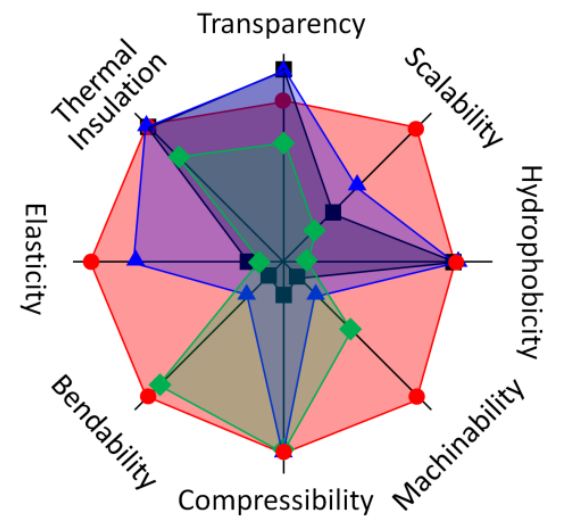

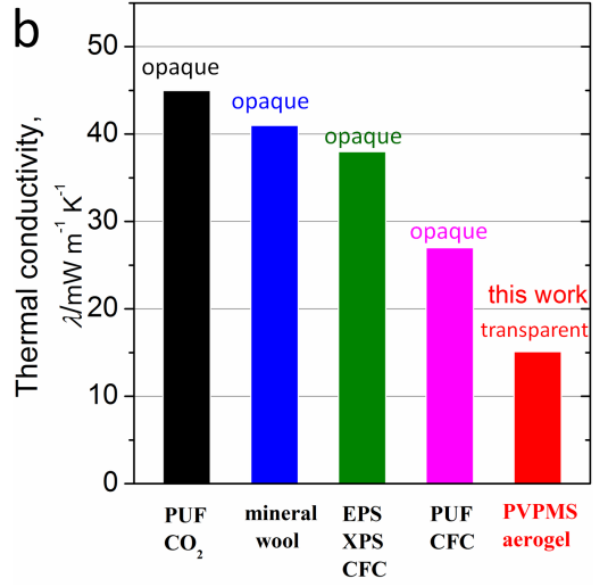

PUF: Polyurethane foam

EPS,XPS: Expanded and extruded polystyrene CFC: Chlorohydrofluorocarbon

$\longrightarrow$ Silica aerogel (APD)

$\longrightarrow$ PMSQ aerogel (APD)

$\longrightarrow$ Nanocellulose aerogel (SCD)

$\longrightarrow$ PVPMS aerogel (APD) (this work)

Figure 5. Comparison of our PVPMS aerogels and other thermal insulation materials. (a) Comparison of the thermal conductivity values and flexibility of our PVPMS aerogels and other reported transparent aerogels: silica aerogel, ${ }^{41}$ PMSQ aerogel, ${ }^{22,47}$ polyvinylsilsesquioxane (PVSQ) aerogel, ${ }^{23}$ nanocellulose aerogel, ${ }^{6}$ and chitosan aerogel. ${ }^{48}$ (b) Comparison of the 
thermal conductivity values of PVPMS aerogels and commercial thermal insulation materials. ${ }^{3,4}$ (c) Comparison of typical properties of PVPMS aerogels and other typical transparent aerogels. All the thermal conductivity values were obtained at room temperature and ambient pressure. The PVPMS aerogels exhibit similar thermal conductivity yet higher scalability and much better flexibility compared to those of the reported transparent highly insulating aerogels. The thermal insulation performance of PVPMS aerogels is also much better than those of the commercial thermal insulation materials.

As expected, the measured thermal conductivity of SCD aerogels SH1-48-1 and SH1-48-2 at room temperature is as low as 15.3 and $15.2 \mathrm{~mW} \mathrm{~m}^{-1} \mathrm{~K}^{-1}$ (Table 1), respectively, which is much lower than those of commercial thermal insulation materials such as mineral wool and polyurethane foam (PUF) ${ }^{4}$ and comparable to those of traditional silica aerogels (14-25 $\mathrm{mW} \mathrm{m}^{-1}$ $\left.\mathrm{K}^{-1}\right)^{41,46}$ and PMSQ aerogels $\left(15-35 \mathrm{~mW} \mathrm{~m}^{-1} \mathrm{~K}^{-1}\right),{ }^{22,47}$ showing a superinsulating performance. The APD xerogels AH1-48-1-100, AH1-48-1-100-IPA and AH5-48-1-100-IPA also show low thermal conductivity of $15.4,15.4$ and $15.2 \mathrm{~mW} \mathrm{~m}^{-1} \mathrm{~K}^{-1}$, respectively, which is almost the same as those of the corresponding SCD aerogels. The comparison of typical properties of our PVPMS aerogels and the reported transparent aerogels and commercial thermal insulation materials is additionally provided in Figure 5. The combination of high scalability, high transparency, high hydrophobicity, superflexibility in compression and bending, excellent machinability and superinsulating properties has not been observed in traditional aerogels and other porous materials. These merits together with the ultralow-cost APD will contribute to their practical 
applications in transparent flexible superinsulators.

Recently, we have also prepared transparent PVPMS aerogel-like xerogels by using IPA as the solvent during the hydrolytic polycondensation process followed by ultralow-cost APD without any solvent exchange and modification. A xerogel with low density of $0.24 \mathrm{~g} \mathrm{~cm}^{-3}$, a high SSA of $796 \mathrm{~m}^{2} \mathrm{~g}^{-1}$ and a high visible-light transmittance of $75 \%$ (for 2-mm thick sample) has been obtained via this method. Since solvent exchange and modification are totally avoided during the synthesis process, their production cost will be further reduced. Besides, this double crosslinking approach is highly extendable. In addition to PVPMS aerogels and xerogels, other kinds of transparent, highly flexible, doubly crosslinked organic-inorganic hybrid aerogels and xerogels have also been synthesized from other organoalkoxysilanes with alkene groups via the same approach. These works will be published elsewhere.

\section{CONCLUSION}

In summary, transparent superflexible doubly crosslinked aerogels have been prepared for the first time from a single precursor-VMDMS or VMDES by radical polymerization followed by hydrolytic polycondensation combined with the ultralow-cost, highly scalable APD technique directly using alcohol as a drying medium. Macroscopic phase separation between the hydrophobic condensates and polar solvent is effectively suppressed due to the high conversion and degree of polymerization of PVMDMS or PVMDES and the accelerated hydrolytic polycondensation. The resulting aerogels and xerogels show a unique doubly crosslinked nanostructure with elastic polymethylsiloxanes crosslinked with flexible hydrocarbon chains, 
leading to an unprecedented combination of low density $\left(0.16-0.22 \mathrm{~g} \mathrm{~cm}^{-3}\right)$, uniform pore size (mainly $<60 \mathrm{~nm}$ ), high SSA (900-1000 $\mathrm{m}^{2} \mathrm{~g}^{-1}$ ), high hydrophobicity ( $>130^{\circ}$ contact angle of water), high transparency ( $>80 \%$ transmittance), excellent machinability, superflexibility in compression (endure 80\% compression strain with 500 cycles), superflexibility in bending (endure 100 bending cycles) and superinsulating properties $\left(\lambda=15.0-15.4 \mathrm{~mW} \mathrm{~m}^{-1} \mathrm{~K}^{-1}\right)$. To the best of our knowledge, this is the first time to achieve these merits in a single aerogel and xerogel. This highly extendable double crosslinking method and these outstanding results will offer a significant progress of porous materials and makes the practical applications of transparent flexible aerogel-based superinsulators feasible.

\section{METHODS}

Materials. DTBP was purchased from Tokyo Chemical Industry Co., Ltd. (Japan). Distilled water was purchased from Hayashi Pure Chemical Ind., Ltd. (Japan). VMDMS, VMDES and TMAOH (25wt\% in $\mathrm{H}_{2} \mathrm{O}$ ) were obtained from Sigma-Aldrich, Co. (USA). Benzyl alcohol (BzOH), IPA and $n$-hexane were purchased from Kishida Chemical Co., Ltd. (Japan). All of the chemical reagents were used as received.

Sample preparation. Given amounts of VMDMS (or VMDES) and DTBP (1 or 5 mol\%) were charged in a hydrothermal reactor. The space above the precursor solution was flushed with $\mathrm{Ar}$ and then the reactor was sealed, after which the whole reactor was heated at $120^{\circ} \mathrm{C}$ for predetermined duration ( 6 h, 12 h, 24 h, 48 h, or 72 h), followed by cooling naturally at room 
temperature, affording a transparent and viscous liquid, mainly containing PVMDMS (or PVMDES). To the solution were added $\mathrm{BzOH}, \mathrm{H}_{2} \mathrm{O}$ and base catalyst (TMAOH) with specific molar ratio under stirring in the listed order. After stirring for 5 min, the sol was transferred into a container, which was then sealed and placed in an oven at $80^{\circ} \mathrm{C}$, where the gel formed within $1 \mathrm{~h}$. The gel was aged at 80 or $100{ }^{\circ} \mathrm{C}$ for 4 or 5 d (the gels of AH1-48-2-100-IPA, AH5-48-2-100-IPA and AH1-48-2-100-IPA-E were aged for 5 d, while other gels were aged for 4 d) and subjected to solvent exchange with IPA at $60{ }^{\circ} \mathrm{C}$ for 3 times (each 8 h) to remove the residual chemicals.

For supercritical drying, the gel was dried from supercritical $\mathrm{CO}_{2}$ at $80{ }^{\circ} \mathrm{C}, 13.5 \mathrm{MPa}$ to afford PVPMS aerogels. For APD from IPA, the gel was slowly dried by evaporation at room temperature for $2-5 \mathrm{~d}$ and at $80{ }^{\circ} \mathrm{C}$ for $4 \mathrm{~h}$ to obtain xerogels. For APD from $n$-hexane, the gel was subjected to solvent exchange with $n$-hexane at $50{ }^{\circ} \mathrm{C}$ for 3 times (each $8 \mathrm{~h}$ ) followed by evaporation at room temperature for $2-3 \mathrm{~d}$ and at $80^{\circ} \mathrm{C}$ for $4 \mathrm{~h}$ to obtain xerogels.

Characterizations. The weight-average molecular weight $\left(M_{\mathrm{w}}\right)$ and polydispersity $\left(M_{\mathrm{w}} / M_{\mathrm{n}}\right)$ of PVMDMS were analyzed by a gel permeation chromatography (GPC) system (GPC104, Shodex, Japan) with a LF604 column and chloroform solvent. The conversion of PVMDMS was calculated from the ${ }^{1} \mathrm{H}$ nuclear magnetic resonance (NMR) spectra measured with a NMR spectrometer (Avance III, Bruker Corp., Germany) operating at $400 \mathrm{MHz}$. The bulk density of aerogels and xerogels was determined by measuring the diameter, height and mass of the cylindrical samples. The skeletal density was determined by Helium pycnometry with an 
automatic density analyzer (ULTRAPYC 1200e, Quantachrome Instruments, USA). The total pore volume was calculated from bulk density and skeletal density. The visible-light transmittance of aerogels and xerogels was measured using a UV-vis-NIR spectrophotometer (V-670, JASCO, Japan) equipped with an integrating sphere.

The surface functional groups of aerogels and xerogels were characterized by a Fourier transform infrared spectroscope (FTIR, IRAffinity-1, Shimadzu Corp., Japan). The pore morphology was observed by a field emission scanning electron microscope (FESEM, JSM-6700F, JEOL, Japan). The $\mathrm{N}_{2}$ adsorption/desorption isotherm, SSA, and pore size distribution were measured by a $\mathrm{N}_{2}$ adsorption analyzer (BELSORP-mini, BEL Japan, Inc., Japan). Before the $\mathrm{N}_{2}$ adsorption/desorption measurement, the aerogel or xerogel sample was degassed at $120^{\circ} \mathrm{C}$ under vacuum for around $6 \mathrm{~h}$. The SSA was obtained from the adsorption branch using the Brunauer-Emmett-Teller (BET) method. The pore size distribution was derived from the adsorption branch using the Barrett-Joyner-Halenda (BJH) calculation.

The uniaxial compression-decompression tests and three-point bending tests were carried out with a material tester (EZGraph, Shimadzu Corp., Japan). The crosshead speed was $0.5 \mathrm{~mm}$ $\min ^{-1}$ for both compression and bending tests. In uniaxial compression-decompression tests, a cylindrical aerogel or xerogel with 8-15 mm diameter and 4-10 $\mathrm{mm}$ height was used. In three-point bending tests, a cuboid aerogel or xerogel with typical width $\times$ length $\times$ height of 10 $\times 50 \times 1 \mathrm{~mm}$ was used. The apparatus span was fixed at $25 \mathrm{~mm}$.

Contact angles of water were measured by Drop Master (DM-561Hi, Kyowa Interface Science Co., Ltd., Japan). The volume of water droplet was fixed at $3 \mu \mathrm{L}$. The thermal stability was 
investigated by thermogravimetric-differential thermal analysis (TG-DTA) with Thermo plus EVO (TG 8120, Rigaku Corp., Japan) at a heating rate of $5{ }^{\circ} \mathrm{C} \min ^{-1}$ in air. The ${ }^{29} \mathrm{Si}$ cross-polarization magic angle spinning (CP/MAS) NMR spectra was obtained from a NMR spectrometer (Avance III 800US Plus, Bruker Corp., Germany), operating at a static magnetic field of $18.8 \mathrm{~T}$. The probe was $4 \mathrm{~mm}$ and the MAS frequency was set to $12 \mathrm{kHz}$ with hexamethylcyclotrisiloxane as an external reference material. The thermal conductivity at room temperature and ambient pressure was determined by a heat flow meter (HFM 436 Lambda, NETZSCH, Germany). A cubic aerogel or xerogel with typical width $\times$ length $\times$ height of $100 \times 100 \times 10 \mathrm{~mm}$ was used for the thermal conductivity measurement.

\section{ASSOCIATED CONTENT}

\section{Supporting Information}

The Supporting Information is available free of charge on the ACS Publications website.

Compression and bending movies showing superflexibility of resulting PVPMS aerogels and xerogels, FTIR spectra, effect of polymerization time and DTBP concentration, hydrophobicity, the porous structure of xerogel AH1-48-1, TG-DTA curves, compressive cycle performances of typical xerogels, structure and bending flexibility of typical xerogel obtained from VMDES, excellent machinability of the PVPMS xerogel, results on the radical polymerization of VMDMS, and compositions and typical properties of aerogels with different polymerization time.

\section{AUTHOR INFORMATION}




\section{Corresponding authors}

*E-mail: guoqingzu@yahoo.com (GZ), kanamori@kuchem.kyoto-u.ac.jp (KK)

\section{Notes}

The authors declare no competing financial interest.

\section{ACKNOWLEDGEMENTS}

Prof. Yasuyuki Nakamura, Dr. Takehiro Fujita and Prof. Shigeru Yamago are acknowledged for their help in GPC and liquid-state NMR measurements. The solid-state NMR measurements were carried out in the JURC at the Institute for Chemical Research, Kyoto University. The present study has been performed under financial supports from Advanced Low Carbon Technology Research and Development Program (ALCA, JST Japan) and JSPS KAKENHI Grant Number JP26288106 and 17K06015.

\section{REFERENCES}

1. Kistler, S. S. Coherent Expanded Aerogels and Jellies. Nature 1931, 127, 741.

2. Kucheyev, S. O.; Stadermann, M.; Shin, S. J.; Satcher Jr., J. H.; Gammon, S. A.; Letts, S. A.; van Buuren, T; Hamza, A. V. Super-compressibility of Ultralow-Density Nanoporous Silica. Adv. Mater. 2012, 24, 776-780.

3. Husing, N; Schubert, U. Aerogels-Airy Materials: Chemistry, Structure, and Properties. Angew. Chem. Int. Ed. 1998, 37, 22-45.

4. Pierre, A. C; Pajonk, G. M. Chemistry of Aerogels and Their Applications. Chem. Rev. 2002, 
102, 4243-4265.

5. Mohanan, J. L.; Arachchige, I. U.; Brock, S. L. Porous Semiconductor Chalcogenide Aerogels. Science 2015, 307, 397-400.

6. Kobayashi, Y.; Saito, T.; Isogai, A. Aerogels with 3D Ordered Nanofiber Skeletons of Liquid-Crystalline Nanocellulose Derivatives as Tough and Transparent Insulators. Angew. Chem. Int. Ed. 2014, 53, 10394-10397.

7. Hees, T.; Zhong, F.; Rudolph, T.; Walther, A.; Mulhaupt, R. Nanocellulose Aerogels for Supporting Iron Catalysts and in situ Formation of Polyethylene Nanocomposites. Adv. Funct. Mater. 2017, 27, 1605586-1605593.

8. Khan, Z. U.; Edberg, J.; Hamedi, M. M.; Gabrielsson, R.; Granberg, H.; Wagberg, L.;

Engquist, I.; Berggren, M.; Crispin, X. Thermoelectric Polymers and Their Elastic Aerogels. Adv. Mater. 2016, 28, 4556-4562.

9. Kim, C. H. J.; Zhao, D.; Lee, G.; Liu, J. Strong, Machinable Carbon Aerogels for High Performance Supercapacitors. Adv. Funct. Mater. 2016, 26, 4976-4983.

10. Aliev, A. E.; Oh, J.; Kozlov, M. E.; Kuznetsov, A. A.; Fang, S.; Fonseca, A. F.; Ovalle, R.; Lima, M. D.; Haque, M. H.; Gartstein, Y. N.; Zhang, M.; Zakhidov, A. A.; Baughman, R. H. Giant-Stroke, Superelastic Carbon Nanotube Aerogel Muscles. Science 2009, 323, 1575-1578.

11. Lin, Z.; Zeng, Z.; Gui, X.; Tang, Z.; Zou, M.; Cao, A. Carbon Nanotube Sponges, Aerogels, and Hierarchical Composites: Synthesis, Properties, and Energy Applications. Adv. Energy Mater. 2016, 6, 1600554-1600579. 
12. Yang, C.; Liu, N.; Zeng, W.; Long, F.; Song, Z.; Su, J.; Li, L.; Zou, Z.; Fang, G.; Xiong, L.; Gao, Y. Superelastic and Ultralight Electron Source from Modifying 3D Reduced Graphene Aerogel Microstructure. Nano Energy 2017, 33, 280-287.

13. Li, C.; Qiu, L.; Zhang, B.; Li, D.; Liu, C. -Y. Robust Vacuum-/Air-Dried Graphene Aerogels and Fast Recoverable Shape-Memory Hybrid Foams. Adv. Mater. 2016, 28, 1510-1516.

14. Toivonen, M. S.; Kaskela, A.; Rojas, O. J.; Kauppinen, E. I.; Ikkala, O. Ambient-Dried Cellulose Nanofibril Aerogel Membranes with High Tensile Strength and Their Use for Aerosol Collection and Templates for Transparent, Flexible Devices. Adv. Funct. Mater. 2015, 25, 6618-6626.

15. Si, Y.; Fu, Q.; Wang, X.; Zhu, J.; Yu, J.; Sun, G.; Ding, B. Superelastic and Superhydrophobic Nanofiber-Assembled Cellular Aerogels for Effective Separation of Oil/Water Emulsions. ACS Nano 2015, 9, 3791-3799.

16. Prakash, S. S.; Brinker, C. J.; Hurd, A. J.; Rao, S. M. Silica Aerogel Films Prepared at Ambient Pressure by Using Surface Derivatization to Induce Reversible Drying Shrinkage. Nature 1995, 374, 439-443.

17. Jiang, L.; Kato, K.; Mayumi, K.; Yokoyama, H.; Ito, K. One-Pot Synthesis and Characterization of Polyrotaxane-Silica Hybrid Aerogel. ACS Macro Lett. 2017, 6, 281-286.

18. Leventis, N. Three-Dimensional Core-Shell Superstructures: Mechanically Strong Aerogels. Acc. Chem. Res. 2007, 40, 874-884.

19. Randall, J. P.; Meador, M. A. B.; Jana, S. C. Tailoring Mechanical Properties of Aerogels for Aerospace Applications. ACS Appl. Mater. Interfaces 2011, 3, 613-626. 
20. Maleki, H.; Duraes, L.; Portugal, A. Synthesis of Mechanically Reinforced Silica Aerogels via Surface-Initiated Reversible Addition Fragmentation Chain Transfer (RAFT) Polymerization. J. Mater. Chem. A 2015, 3, 1594-1600.

21. Kanamori, K.; Aizawa, M.; Nakanishi, K.; Hanada, T. New Transparent Methylsilsesquioxane Aerogels and Xerogels with Improved Mechanical Properties. Adv. Mater. 2007, 19, 1589-1593.

22. Hayase, G.; Kugimiya, K.; Ogawa, M.; Kodera, Y.; Kanamori, K.; Nakanishi, K. The Thermal Conductivity of Polymethylsilsesquioxane Aerogels and Xerogels with Varied Pore Sizes for Practical Application as Thermal Superinsulators. J. Mater. Chem. A 2014, 2, 6525-6531.

23. Shimizu, T.; Kanamori, K.; Maeno, A.; Kaji, H.; Doherty, C. M.; Falcaro, P.; Nakanishi, K. Transparent, Highly Insulating Polyethyl- and Polyvinylsilsesquioxane Aerogels: Mechanical Improvements by Vulcanization for Ambient Pressure Drying. Chem. Mater. 2016, 28, 6860-6868.

24. Hayase, G.; Kanamori, K.; Fukuchi, M.; Kaji, H.; Nakanishi, K. Facile Synthesis of Marshmallow-Like Macroporous Gels Usable Under Harsh Conditions for the Separation of Oil and Water. Angew. Chem. Int. Ed. 2013, 52, 1986-1989.

25. Wang, Z.; Dai, Z.; Wu, J.; Zhao, N.; Xu, J. Vacuum-Dried Robust Bridged Silsesquioxane Aerogels. Adv. Mater. 2013, 25, 4494-4497.

26. Yun, S.; Luo, H.; Gao, Y. Low-Density, Hydrophobic, Highly Flexible Ambient Pressure-Dried Monolithic Bridged Silsesquioxane Aerogels. J. Mater. Chem. A 2015, 3, 
3390-3398.

27. Shimizu, T.; Kanamori, K.; Maeno, A.; Kaji, H.; Nakanishi, K. Transparent Ethylene-Bridged Polymethylsiloxane Aerogels and Xerogels with Improved Bending Flexibility. Langmuir 2016, 32, 13427-13434.

28. Zou, F.; Yue, P.; Zheng, X.; Tang, D.; Fu, W.; Li, Z. Robust and Superhydrophobic Thiourethane Bridged Polysilsesquioxane Aerogels as Potential Thermal Insulation Materials. J. Mater. Chem. A 2016, 4, 10801-10805.

29. Aoki, Y.; Shimizu, T.; Kanamori, K.; Maeno, A.; Kaji, H.; Nakanishi, K. Low-Density, Transparent Aerogels and Xerogels Based on Hexylene-Bridged Polysilsesquioxane with Bendability. J. Sol-Gel. Sci. Technol. 2017, 81, 42-51.

30. Shimizu, T.; Kanamori, K.; Maeno, A.; Kaji, H.; Doherty, C. M.; Nakanishi, K. Transparent Ethenylene-Bridged Polymethylsiloxane Aerogels: Mechanical Flexibility and Strength and Availability for Addition Reaction. Langmuir 2017, 33, 4543-4550.

31. Nguyen, B. N.; Meador, M. A. B.; Tousley, M. E.; Shonkwiler, B.; McCorkle, L.; Scheiman, D. A.; Palczer, A. Tailoring Elastic Properties of Silica Aerogels Cross-Linked with Polystyrene. ACS Appl. Mater. Interfaces 2009, 1, 621-630.

32. Abe, Y.; Namiki, T.; Tuchida, K.; Nagao, Y.; Misono, T. Preparation and Properties of Silicon-Containing Hybrid Gels From Vinyltrimethoxysilane. J. Non-Cryst. Solids 1992, $147 \& 148,47-51$.

33. Gunji, T.; Kawaguchi, Y.; Okonogi, H.; Sakan, T.; Arimitsu, K.; Abe, Y. Preparation and Properties of Organic-Inorganic Hybrid Gel Films Based on Polyvinylpolysilsesquioxane 
Synthesized from Trimethoxy(vinyl)silane. J. Sol-Gel Sci. Technol.2005, 33, 9-13.

34. Gong, J. P.; Katsuyama, Y.; Kurokawa, T.; Osada, Y. Double-Network Hydrogels with Extremely High Mechanical Strength. Adv. Mater. 2003, 15, 1155-1158.

35. Nonoyama, T.; Wada, S.; Kiyama, R.; Kitamura, N.; Mredha, M. T. I.; Zhang, X.; Kurokawa, T.; Nakajima, T.; Takagi, Y.; Yasuda, K.; Gong, J. P. Double-Network Hydrogels Strongly Bondable to Bones by Spontaneous Osteogenesis Penetration. Adv. Mater. 2016, 28, 6740-6745.

36. Zhao, Y.; Nakajima, T.; Yang, J. J.; Kurokawa, T.; Liu, J.; Lu, J.; Mizumoto, S.; Sugahara, K.; Kitamura, N.; Yasuda, K.; Daniels, A. U. D.; Gong, J. P. Proteoglycans and Glycosaminoglycans Improve Toughness of Biocompatible Double Network Hydrogels. Adv. Mater. 2014, 26, 436-442.

37. Zhang, H. J.; Sun, T. L.; Zhang, A. K.; Ikura, Y.; Nakajima, T.; Nonoyama, T.; Kurokawa, T.; Ito, O.; Ishitobi, H.; Gong, J. P. Tough Physical Double-Network Hydrogels Based on Amphiphilic Triblock Copolymers. Adv. Mater. 2016, 28, 4884-4890.

38. Hayase, G.; Kanamori, K.; Hasegawa, G.; Maeno, A.; Kaji, H.; Nakanishi, K. A Superamphiphobic Macroporous Silicone Monolith with Marshmallow-Like Flexibility. Angew. Chem. Int. Ed. 2013, 52, 10788-10791.

39. Hong, J. Y.; Bak, B. M.; Wie, J. J.; Kong, J.; Park, H. S. Reversibly Compressible, Highly Elastic, and Durable Graphene Aerogels for Energy Storage Devices Under Limiting Conditions. Adv. Funct. Mater. 2015, 25, 1053-1062.

40. Moitra, N.; Ichii, S.; Kamei, T.; Kanamori, K.; Zhu, Y.; Takeda, K.; Nakanishi, K.; Shimada, 
T. Surface Functionalization of Silica by Si-H Activation of Hydrosilanes. J. Am. Chem. Soc. 2014, 136, 11570-11573.

41. Wong, J. C. H.; Kaymak, H.; Brunner, S.; Koebel, M. M. Mechanical Properties of Monolithic Silica Aerogels Made from Polyethoxydisiloxanes. Micropor. Mesopor. Mater. 2014, 183, 23-29.

42. Zu, G.; Shen, J.; Wang, W.; Zou, L.; Lian, Y.; Zhang, Z.; Liu, B.; Zhang, F. Robust, Highly Thermally Stable, Core-Shell Nanostructured Metal Oxide Aerogels as High-Temperature Thermal Superinsulators, Adsorbents, and Catalysts. Chem. Mater. 2014, 26, 5761-5772.

43. Ma, C. -B.; Du, B.; Wang, E. Self-Crosslink Method for a Straightforward Synthesis of Poly(vinyl alcohol)-Based Aerogel Assisted by Carbon Nanotube. Adv. Funct. Mater. 2017, 27, 1604423-1604431.

44. Hasegawa, G.; Shimizu, T.; Kanamori, K.; Maeno, A.; Kaji, H.; Nakanishi, K. Highly Flexible Hybrid Polymer Aerogels and Xerogels Based on Resorcinol-Formaldehyde with Enhanced Elastic Stiffness and Recoverability: Insights into the Origin of Their Mechanical Properties. Chem. Mater. 2017, 29, 2122-2134.

45. Lu, X.; Arduini-Schuster, M. C.; Kuhn, J.; Nilsson, O.; Fricke, J.; Pekala, R. W. Thermal Conductivity of Monolithic Organic Aerogels. Science 1992, 255, 971-972.

46. Huber, L.; Zhao, S.; Malfait, W. J.; Vares, S.; Koebel, M. M. Fast and Minimal-Solvent Production of Superinsulating Silica Aerogel Granulate. Angew. Chem. Int. Ed. 2017, 56, 4753-4756.

47. Hayase, G.; Kanamori, K.; Abe, K.; Yano, H.; Maeno, A.; Kaji, H.; Nakanishi, K. 
Polymethylsilsesquioxane-Cellulose Nanofiber Biocomposite Aerogels with High Thermal

Insulation, Bendability, and Superhydrophobicity. ACS Appl. Mater. Interfaces 2014, 6, 9466-9471.

48. Takeshita, S.; Yoda, S. Chitosan Aerogels: Transparent, Flexible Thermal Insulators. Chem.

Mater. 2015, 27, 7569-7572. 